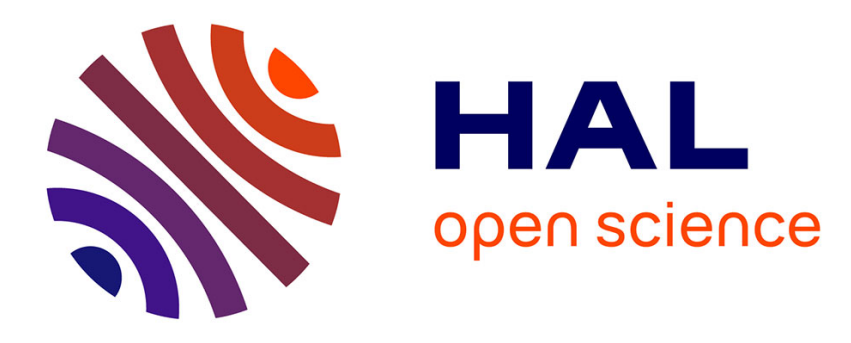

\title{
Manifestations de la soft law en droit français des religions
}

\author{
Françoise Curtit, Anne Fornerod
}

\section{To cite this version:}

Françoise Curtit, Anne Fornerod. Manifestations de la soft law en droit français des religions. Studies in Religion/Sciences Religieuses, 2016, 45 (2), pp.111 - 126. 10.1177/0008429816636083 . halshs01658018

\section{HAL Id: halshs-01658018 \\ https://shs.hal.science/halshs-01658018}

Submitted on 7 Dec 2017

HAL is a multi-disciplinary open access archive for the deposit and dissemination of scientific research documents, whether they are published or not. The documents may come from teaching and research institutions in France or abroad, or from public or private research centers.
L'archive ouverte pluridisciplinaire HAL, est destinée au dépôt et à la diffusion de documents scientifiques de niveau recherche, publiés ou non, émanant des établissements d'enseignement et de recherche français ou étrangers, des laboratoires publics ou privés. 


\title{
Manifestations de la soft law en droit français des religions
}

\author{
Françoise Curtit \\ DRES - Droits et religions, CNRS ; Université de Strasbourg, France \\ Anne Fornerod \\ DRES - Droits et religions, CNRS ; Université de Strasbourg, France
}

Résumé : Constitué d'une part importante de textes de type infra-réglementaires et nourri d'adaptations au contexte contemporain d'une législation ancienne, le droit des religions est un lieu d'incarnation de la soft law entendue notamment comme l'usage de la négociation en marge du recours à la loi. Le régime applicable au patrimoine religieux est un parfait exemple de construction juridique bâtie au fil des contingences historiques et d'aménagements successifs et la valorisation du patrimoine religieux mobilise aujourd'hui ces mécanismes d'élaboration d'un droit marqué par le pragmatisme et la flexibilité.

\begin{abstract}
:
The legal framework applicable to religions is composed of many infra-administrative regulations and shaped by the adaptations of old legislative norms to the contemporary context. It results that law and religion fall within the scope of a certain conception of soft law, such as resorting to negotiation in the margin of legislative texts. The issue of religious heritage perfectly illustrates the process which consists in building and updating a legal status over historical, social and political circumstances. The enhancement of religious heritage in particular rests on mechanisms typical of soft law.
\end{abstract}

\section{Mots clés}

droit des religions, soft law, édifices du culte, patrimoine religieux

\section{Keywords}

law and religion, soft law, worship buildings, religious heritage

Studies in Religion / Sciences Religieuses 2016

Vol. 45(2) 111-126

DOI: $10.1177 / 0008429816636083$ 
$\mathrm{Si}$, au cours des dernières décennies, les questions liées à la religion ont alimenté le débat public et donné lieu à une jurisprudence de plus en plus abondante, l'appréhension du fait religieux ${ }^{1}$ par le droit a en France des racines historiques anciennes (Messner et al., $2013: 195$ et s.). Succédant au droit ecclésiastique d'Ancien Régime, le droit des cultes se développe après la Révolution française d'une part à partir d'un corpus de normes législatives et réglementaires qui visent à encadrer les relations entre l'État et les institutions religieuses et, d'autre part, à partir d'une jurisprudence administrative qui intervient principalement en matière d'organisation des confessions « reconnues ». Ces règles, fortement ancrées dans un contexte historique et politique, composent le socle du «droit des religions » tel qu'il s'applique aujourd'hui encore, alors que le paysage religieux de la France s'est profondément transformé. Les dispositions qui le constituent ont fait l'objet au fil des époques d'ajustements unilatéraux ou négociés qui ont permis de les adapter à un contexte sociologique et institutionnel fort différent de celui de leur création. Est-ce le résultat d'une spécificité de ce droit, liée notamment à son objet ? La proximité - ou la présence - de la laïcité dans l'environnement politique, idéologique, mais aussi juridique de ces questions expliquerait-elle la structure du droit des religions ? En effet, la charge symbolique qui accompagne le fait religieux explique certaines réticences du législateur à intervenir et se manifeste notamment par la part prépondérante des sources réglementaires en la matière. L'hypothèse d'une telle spécificité mérite d'être complétée par la référence à un phénomène qui affecte quasiment l'ensemble des branches du droit, à savoir le développement de la soft law. L'absence même de consensus au sujet de la traduction de cette notion issue du droit international révèle la difficulté de saisir dans son entier ce que l'on entend par soft law². À l'instar du « droit dur » classique, la soft law se compose de règles de conduite qui visent à encadrer les comportements sociaux. En revanche, elle s'en distingue par son mode de formulation et son contenu : elle incite plus qu'elle n'impose. II n'en demeure pas moins entendu que ce droit, ou plutôt cette façon d'édicter des normes juridiques, est révélatrice des transformations profondes du droit luimême et des modes d'action de l'État et des institutions publiques. À la contrainte unilatérale sont préférées la discussion et la recherche d'une adhésion à la norme (Thibierge, 2003). L'adaptabilité du droit des religions au paysage religieux contemporain lui est-elle consubstantielle et peut-on y voir un terrain d'application de la soft law? S'il paraît difficile de répondre définitivement à cette question, 
force est de constater que le droit des religions, loin d'être unilatéral, repose sur une volonté des pouvoirs publics d'ajuster la norme aux besoins des acteurs, voire, à l'occasion, résulte d'une négociation entre les parties en présence (1). Tel est le cas de la gestion du patrimoine religieux, dont il sera ici question à titre illustratif (2).

\section{Le fait religieux saisi par le droit : un encadrement des organisations confessionnelles entre ajustements et adaptations}

Un droit des organisations confessionnelles plutôt qu'un droit de la liberté religieuse

Le fait religieux est régulé aujourd'hui par un ensemble de dispositions issues de plusieurs périodes historiques qui chacune ont imprimé leur marque. Dans les années 1880 sont votées les grandes lois de la III ${ }^{\mathrm{e}}$ République (laïcité de l'enseignement, neutralité des cimetières, liberté de réunion, laïcisation des funérailles...), puis au tournant $d u X X^{e}$ siècle la loi de séparation fixe le cadre juridique réglementant l'organisation des associations religieuses et de leurs personnels, la police du culte et la gestion des édifices cultuels. Ce corpus sera complété au cours de la deuxième moitié du $X X^{e}$ siècle pour tenir compte de nouveaux domaines d'intervention qui, cette fois encore, mettent en présence pouvoirs publics et organisations confessionnelles (enseignement privé, protection sociale des ministres du culte, abattage rituel...). À l'exception de quelques interventions législatives emblématiques adoptées dans les années $2000^{3}$, le droit français des religions repose aujourd'hui sur cet héritage juridique relativement ancien, modifié par touches successives au cours des décennies, mais sans que son économie générale ait été notablement modifiée.

Une analyse quantitative ${ }^{4}$ des textes qui évoquent le fait religieux sous ses divers aspects révèle qu'une part prépondérante de ceux-ci concerne l'organisation des cultes sur le plan institutionnel (statut des associations et congrégations, droit fiscal, organisation des services d'aumôneries, personnel des institutions religieuses). Un nombre important de dispositions encadrent par ailleurs l'intervention des pouvoirs publics dans l'organisation matérielle de l'exercice du culte (police des cultes, lieux de culte, abattage rituel, funérailles et inhumations) ainsi que, dans une moindre mesure, l'organisation de l'enseignement (laïcité dans l'enseignement public, aumôneries scolaires, 
établissements d'enseignement privés). En dernier lieu, un petit nombre de dispositions éparses vont garantir la liberté de conscience et le principe de non-discrimination ${ }^{5}$. Ce rapide inventaire fait apparaître qu'au prisme des textes juridiques, la religion est appréhendée essentiellement dans sa dimension institutionnelle: il s'agit avant tout d'administrer et de financer ${ }^{6}$ des organisations confessionnelles, leurs bâtiments et leurs personnels et de régler leurs rapports avec les pouvoirs publics. Les normes juridiques dessinent les contours d'un droit des organisations cultuelles plutôt qu'un droit des pratiques religieuses. Le fait religieux paraît régulé sous l'angle des relations entre institutions plutôt qu'entre d'individus et apparaît comme une affaire de gestion plutôt que de convictions. II est d'ailleurs symptomatique que la loi du 9 décembre 1905 concernant la séparation des Églises et de l'État, perçue aujourd'hui comme le socle fondateur de la laïcité en France, soit consacrée pour l'essentiel à la propriété des biens ecclésiastiques et des édifices cultuels, aux pensions des ministres du culte, à la création d'associations à objet cultuel et à des règles de police administrative. La force symbolique des deux premiers articles de la $\mathrm{loi}^{7}$ qui proclament liberté de conscience, libre exercice des cultes et interdiction des subventions publiques a éclipsé dans l'imaginaire collectif la fonction première de ce texte qui visait à mettre en place un nouveau mode de gestion des organisations et du patrimoine religieux, ces règles conservant aujourd'hui l'essentiel de leur portée juridique.

Si la voie législative a été réactivée récemment à propos de la visibilité des signes religieux à l'école $(15 \text { mars } 2004)^{8}$ et dans l'espace public (11 octobre 2010$)^{9}$, c'est pour adopter en la matière une approche prohibitive, le principe de laïcité et la défense de l'ordre public primant ici sur la liberté d'expression des convictions religieuses ${ }^{10}$. C'est précisément sur cette question des manifestations de la liberté de religion que le droit français montre ses limites. La garantie de liberté de conscience et de libre exercice du culte est énoncée dans plusieurs textes ${ }^{11}$. Pour autant, le principe de liberté de religion, s'il est encadré par le droit international et clairement garanti par la jurisprudence nationale, n'est pas énoncé en tant que tel dans la Constitution de la $V^{\text {ème }}$ République (Messner et al., 2013 : 654), contrairement à ce que prévoient les lois fondamentales de la plupart des autres pays européens. Détrônée sur le plan tant symbolique que juridique par le rôle prépondérant accordé au principe de laïcité, la garantie des droits fondamentaux, et en particulier de la liberté de religion, 
semble en quelque sorte déconnectée du corpus des dispositions qui réglementent les organisations et activités cultuelles.

Ce laconisme des textes au sujet de la liberté de religion s'explique notamment par la structure même du droit français des religions constitué d'un réseau de normes parcourant les diverses branches du droit (droit constitutionnel, droit administratif, droit fiscal, droit de l'urbanisme, droit pénal...) en agrégeant et superposant règles de droit commun et règles propres aux institutions et activités cultuelles, sans qu'elles émanent d'un principe général de valeur supra législative. Ce maillage de normes est d'ailleurs à mettre en relation avec la nature des dispositions concernées : interprété comme un ensemble d'organisations, de bâtiments et de personnels dont il s'agit d'encadrer l'administration et les rapports avec les pouvoirs publics, le fait religieux est régulé par un droit essentiellement réglementaire, un droit pragmatique qui, s'il est constitué de dispositions souvent anciennes, n'en est pas moins facilement adaptable aux pratiques et institutions existantes.

\section{Un droit qui s'ajuste aux pratiques et institutions existantes}

S'il a fait un retour remarqué dans les années 2000 comme reflet d'une volonté politique d'affirmation de valeurs, le recours à la loi en matière proprement religieuse n'était plus guère de mise depuis les années $1950^{12}$. Le droit des religions tel qu'il s'applique aujourd'hui reste un droit de nature essentiellement réglementaire réunissant décrets, arrêtés et circulaires. II faut d'emblée souligner que cette dernière catégorie de textes occupe une place particulièrement importante au sein du corpus de normes considéré, près de quarante circulaires rédigées depuis la fin des années 1960 étant aujourd'hui d'application ${ }^{13}$. Si certaines d'entre elles visent assez classiquement à expliciter et faciliter l'application d'un texte donné ${ }^{14}$, beaucoup s'efforcent plutôt de "codifier » le droit en vigueur en rappelant le régime juridique applicable à la lumière des évolutions de la jurisprudence ${ }^{15}$, voire en réaction à " des évènements récents ${ }^{16}$. II s'agit de clarifier les principes et règles existants sur des sujets qui font l'objet d'une réglementation complexe et insuffisamment adaptée aux situations actuelles (régime de propriété et d'affectation des édifices du culte ${ }^{17}$, régime juridique des associations ${ }^{18} \ldots$ ) ou qui suscitent débats ou tensions sur le terrain et pour lesquels il n'existe pas de dispositions normatives suffisamment précises (laïcité dans les établissements de santé ${ }^{19}$, régimes 
alimentaires et restauration collective des services publics ${ }^{20} \ldots$ ). Instrument destiné aux autorités et services administratifs, la circulaire constitue ainsi un outil souple et rapide d'actualisation du droit en matière religieuse, mais aussi d'ajustement voire d'entérinement de la pratique administrative sur des questions auxquelles sont confrontées les acteurs locaux et pour lesquelles les normes juridiques traditionnelles n'offrent pas de solutions satisfaisantes.

Ainsi, en matière de sépultures, la loi du 14 novembre 1881 a supprimé la possibilité d'établir au sein des cimetières des séparations en fonction des différents cultes ${ }^{21}$. En 1975 cependant, une circulaire du ministère de l'Intérieur ${ }^{22}$ permettait une dérogation à ce principe en recommandant aux maires de « réserver aux Français de confession islamique [...] des carrés spéciaux dans les cimetières existants ", afin d'apporter "une solution particulière au problème de l'inhumation de nos compatriotes musulmans $»^{23}$. La circulaire du 19 février $2008^{24}$ en vigueur aujourd'hui les « encourage ${ }^{25}$ dans le même esprit « à favoriser [...] l'existence d'espaces regroupant les défunts de même confession », car "si le principe de laïcité des lieux publics, en particulier des cimetières, doit être clairement affirmé, il apparaît souhaitable, par souci d'intégration des familles issues de l'immigration, de favoriser l'inhumation de leurs proches sur le territoire français ». L'on est face d'une part à une norme de valeur législative datant de la fin du $\mathrm{XIX}^{\mathrm{e}}$ siècle qui affirme la neutralité des cimetières et, d'autre part, à une succession de circulaires $(1975,1991,2008)$ relevant du domaine de l'infra-droit ${ }^{26}$ qui, si elles ne contiennent pas en la matière de dispositions impératives, demandent aux préfets d'inciter les maires à créer des « carrés confessionnels » dont le statut juridique demeure en tout état de cause très fragile ${ }^{27}$. Plutôt que la voie de la réforme législative qui pourrait être source de controverse idéologique et politique, c'est celle d'un compromis pragmatique qui est ici choisie, les circulaires visant à répondre à des situations concrètes en adaptant la législation aux besoins des acteurs de terrain, sans modifier expressément les normes législatives existantes et sans en créer de nouvelles.

Un grand nombre de dispositions appliquées aujourd'hui sont antérieures aux années 1950 et elles ont dans bien des cas fait l'objet d'interprétations libérales par la jurisprudence ou d'adaptations par voie de circulaires administratives pour répondre aux nouveaux usages nés d'une société religieusement diversifiée. Cette faculté de souplesse et d'adaptabilité du droit des religions semble 
cependant en panne dans un certain nombre de domaines liés notamment à l'expression publique de religions considérées comme minoritaires (construction d'édifices cultuels, port de signes religieux, régimes alimentaires...). Des aménagements pourraient être là aussi mis en œuvre, mais cette faculté paraît contrecarrée par une conception de la laïcité qui s'appuie sur des motifs d'ordre idéologique et politique qui rendent la règle de droit inaudible ${ }^{28}$.

Constitué d'une part importante de textes de type infra-réglementaires, nourri d'adaptations d'une législation ancienne au contexte contemporain, le droit des religions est un lieu d'incarnation d'une conception renouvelée de la soft law, vue ici comme un «moyen prudent pour les autorités administratives de mener leur action, prudence qui témoigne d'une forme d'impuissance lorsqu'elle correspond à un évitement de la contrainte, mais aussi d'un certain pragmatisme dans le choix délibéré de ne pas lier ses destinataires et soi-même » (Lavergne, $2011: 287$ ).

Cette recherche d'une meilleure réception de la règle de droit qui caractérise la soft law s'exprime également en droit des religions par l'usage de la négociation de la norme entre parties en présence, la loi commune pouvant dans certains cas s'accorder aux situations particulières pour dessiner un « droit de régulation » marqué par le pragmatisme et la flexibilité (Chevallier, 2001). Les ajustements de la règle ne concernent pas les seuls cultes d'implantation récente et la négociation de la norme juridique a en particulier été le fait d'organisations religieuses « historiques », et parmi elles de l'Église catholique qui affirmait ainsi sa position dominante dans le paysage religieux français. Confession sociologiquement majoritaire pendant des siècles dans l'Hexagone, l'Église catholique a en effet développé des relations de dialogue et de négociation avec les pouvoirs publics, notamment par l'entremise du Saint-Siège qui dispose d'une personnalité de droit international lui permettant d'engager des relations diplomatiques avec les États. Ainsi, c'est par le moyen de l'encyclique papale Gravissimo officii munere du 10 août 1906 que les autorités catholiques ont refusé d'appliquer les dispositions de la loi du 9 décembre 1905 relatives à la constitution d'associations cultuelles «pour subvenir aux frais, à l'entretien et à l'exercice public d'un culte » (art. 18), associations qu'elles jugeaient non conformes aux règles d'organisation générale du culte catholique et en particulier au principe d'unité du diocèse sous l'autorité de l'évêque ${ }^{29}$. Face à ce refus et pour assurer la continuité de l'exercice du culte, le gouvernement a alors aménagé ces dispositions et fait 
voter les lois du 2 janvier 1907 et 13 avril $1908^{30}$ qui énoncent qu'« à défaut d'associations cultuelles ", les édifices affectés à l'exercice du culte ainsi que les meubles les garnissant sont laissés à la disposition des fidèles et des ministres du culte pour la pratique de leur religion (art. 5 de la loi de 1907) et deviennent la propriété des communes sur le territoire desquelles ils sont situés (art. $1^{\mathrm{er}}$ de la loi de 1908). Le législateur reconnaît par ailleurs la validité d'autres formes d'association pour assurer l'exercice du culte (art. 4 de la loi de 1907) et assouplit ainsi notablement le régime juridique issu de la loi de 1905, accompagné d'ailleurs dans cette volonté d'apaisement par la jurisprudence du Conseil d'État. Cette politique d'ajustements va permettre la reprise des relations diplomatiques entre le gouvernement français et le Saint-Siège et un échange de lettres aboutira en 1924 à un modus vivendi entre les parties prenantes, accord diplomatique en forme simplifiée qui fixe le régime juridique d'associations «diocésaines » selon des statuts-types propres à l'Église catholique (Conseil d'État, $2004: 280-283)$.

Ainsi, des règles dérogatoires sont créées pour la confession majoritaire, issues d'une volonté de conciliation des pouvoirs publics qui souhaitaient répondre à l'opposition de Rome et ont adapté aux principes dogmatiques de l'Église catholique une législation conçue au départ pour s'appliquer de façon indifférenciée à tous les cultes. Le statut de droit international conféré à l'Église catholique a certainement joué un rôle déterminant dans ce processus, mais c'est également sa position d'institution confessionnelle dominante qui lui a valu ce traitement de faveur. Le refus de l'Église catholique de constituer des associations cultuelles a entraîné le transfert de propriété de ses édifices aux communes et lui permet de bénéficier aujourd'hui d'un régime de financement public pour leur entretien et conservation particulièrement favorable au vu de l'ampleur du patrimoine foncier concerné. Cette soustraction à la loi commune a ainsi valu aux catholiques d'obtenir un régime juridique «sur mesure » au final plus avantageux que le régime appliqué aux autres confessions. II s'agit là d'une illustration de l'élaboration de normes juridiques issues de discussions entre parties qui conduisent à adapter la législation à une institution à laquelle on reconnaît un mode de régulation propre (ici les dispositions de droit canonique et l'autorité de l'évêque en matière de dogme et d'administration interne). Le droit s'ajuste à des pratiques et institutions existantes et agit dans le but 
de «perpétuer le passé dans le futur, permettre que ce qui a été continue d'être » (Rivero, 1965 : $683)^{31}$

«Complexe, fragmenté, d'une cohérence parfois incertaine ", le régime applicable aux édifices cultuels (Conseil d'État, 2004 : 299-312) est un exemple de construction juridique bâtie au fil des contingences historiques et d'aménagements successifs et qui repose aujourd'hui encore sur l'organisation et la présence des différents cultes tels qu'ils existaient au début du $X X^{e}$ siècle. Ainsi, quatre régimes de propriété des édifices cultuels coexistent (Flores-Lonjou, 2011) qui déterminent notamment les conditions de leur affectation à un usage religieux. Pour les édifices cultuels catholiques, l'articulation entre les prérogatives du propriétaire et celles de l'affectataire a donné lieu à une abondante jurisprudence du Conseil d'État qui continue de préciser les termes du régime mis en place au moment de la séparation. C'est aujourd'hui le développement d'activités touristiques ou culturelles liées à la mise en valeur du patrimoine religieux - catholique - qui mobilise les mécanismes ainsi exposés d'élaboration du droit des religions, entre surreprésentation des sources réglementaires, négociations en marge du recours à la loi et rôle déterminant de la jurisprudence administrative.

\section{La valorisation du patrimoine religieux : un archétype du droit des religions ?}

En 2004, le Conseil d'État constatait dans son rapport sur la laïcité que « le régime juridique défini en 1905 pour les édifices cultuels existants a été en permanence aménagé et adapté aux exigences de chaque époque, dans un esprit de pragmatisme et dans le respect de ses grands équilibres. Signe des temps, c'est le développement, autour des édifices cultuels, des activités économiques, touristiques ou culturelles, qui appelle aujourd'hui une démarche de même nature. Une clarification des règles applicables paraît en effet nécessaire pour déterminer les droits et devoirs de chacun des acteurs concernés par cette évolution, propriétaires ou affectataires » (Conseil d'État, 2004 : 309-310). Or, cette clarification n'a pas été pleinement réalisée et correspond à un processus encore en cours aujourd'hui. II ne s'agit pas ici d'exposer ou de retracer l'évolution du droit applicable ${ }^{32}$, mais de montrer comment, alors que les textes législatifs existants doivent être adaptés ou complétés, les intérêts divergents rendent difficile la seule intervention étatique unilatérale. 
Cette divergence d'intérêts et la nécessité de s'entendre qui en découle se manifestent doublement. En premier lieu, il résulte du dispositif issu de la séparation que la propriété des édifices du culte catholiques est publique ${ }^{33}$ et que leur usage revient aux autorités religieuses, qui en sont donc les affectataires. À ce titre, elles définissent les modalités des pratiques religieuses qui s'y déroulent ${ }^{34}$. II s'ensuit une cohabitation entre deux types de prérogatives exercées sur un même lieu (propriétaire et affectataire). Ce fut l'œuvre du juge administratif d'en régler les détails au lendemain de la séparation, dans le but de garantir les pratiques religieuses, mais en limitant sensiblement la possibilité pour les personnes publiques de valoriser leur propriété. En second lieu, les édifices du culte sont devenus, quelques décennies plus tard, le siège d'un enjeu supplémentaire : l'accès au patrimoine culturel, dont ils constituent depuis toujours une part considérable. Cette nouvelle problématique s'est non pas substituée, mais ajoutée à celle préexistante de la conciliation entre propriété publique et usage religieux des bâtiments. Sur le terrain de l'entretien des bâtiments, corrélatif du statut de propriétaire, les modalités de relations entre les pouvoirs publics et l'Église catholique sont restées sensiblement les mêmes. En revanche, le statut patrimonial des édifices cultuels est à l'origine de nouvelles revendications, tant en ce qui concerne l'usage que la propriété. Pour le premier, les revendications portent sur l'usage culturel possible de ce patrimoine, concurrent d'une utilisation religieuse déclinante et qui bénéficie pourtant d'une protection légale particulièrement importante. Les revendications de l'État propriétaire renvoient à un autre débat, qui est celui du financement de l'entretien des édifices et du statut même de propriétaire avec les prérogatives qui lui sont traditionnellement attachées, en l'occurrence la possibilité de percevoir une redevance pour toute occupation du domaine public, à l'occasion notamment d'activités culturelles se déroulant dans les édifices du culte catholiques ${ }^{35}$. La priorité - voire l'exclusivité - donnée à l'usage religieux pendant des décennies a en effet réduit d'autant le pouvoir de gestion de l'État et des communes propriétaires et la pleine valorisation de ces lieux. Or, la hausse et la démocratisation de la fréquentation du patrimoine culturel et une baisse notable concomitante des pratiques religieuses rendent nécessaire de définir les conditions d'une cohabitation devenue inévitable pour un certain nombre de lieux.

C'est dans ce contexte qu'ont été adoptées en 2006 les dispositions de l'article L. 2124-31 du Code général de la propriété des personnes publiques (CGPPP) selon lesquelles «lorsque la visite de 
parties d'édifices affectés au culte, notamment de celles où sont exposés des objets mobiliers classés ou inscrits, justifie des modalités particulières d'organisation, leur accès est subordonné à l'accord de I'affectataire. II en va de même en cas d'utilisation de ces édifices pour des activités compatibles avec l'affectation cultuelle. L'accord précise les conditions et les modalités de cet accès ou de cette utilisation. Cet accès ou cette utilisation donne lieu, le cas échéant, au versement d'une redevance domaniale dont le produit peut être partagé entre la collectivité propriétaire et l'affectataire ». Illustrant la complexité de l'ensemble constituant le droit des religions, ce texte a été précédé de circulaires, d'interventions du juge administratif et de négociations. C'est ainsi que l'accord préalable de l'affectataire cultuel à tout usage non religieux des édifices du culte a émergé comme la solution adaptée à la gestion de la valorisation du patrimoine cultuel. Or, postérieurement à l'adoption du texte du code, l'on retrouve à nouveau toutes les « figures » du droit des religions.

\section{Des circulaires à la loi : le principe de l'accord préalable de l'affectataire cultuel}

La nécessité de clarifier la situation résultant du développement des pratiques culturelles dans des édifices affectés légalement au culte s'est fait ressentir relativement tôt, conduisant à retenir comme pivot de cette cohabitation entre pratiques religieuses et fréquentation touristique l'accord préalable de l'affectataire cultuel à tout usage non religieux. Dès 1969, une circulaire du ministre d'État chargé des affaires culturelles fait déjà référence au cas spécifique des cathédrales ${ }^{36}$. Le texte, qui vise à “ déconcentrer " auprès des conservateurs "le pouvoir d'autoriser des manifestations diverses, illuminations saisonnières et tournages de films professionnels dans les monuments de l'État » prend en considération le statut particulier des cathédrales en ce qu'il est précisé que « le pouvoir de délivrer certaines autorisations [...] doit ici s'exercer en concordance avec le pouvoir de réglementation cultuelle que le clergé tient de la législation de Séparation. Pour cette raison, vous ne pouvez dans les cathédrales délivrer d'autorisations que sous réserve de l'accord du Clergé. »

Quelques vingt années plus tard, la circulaire du ministre de la Culture du 27 avril $1988^{37}$ est plus claire et plus favorable aux prérogatives de l'affectataire. La nouvelle procédure prévue a pour principale conséquence de laisser au « clergé, bénéficiant de l'affectation pleine et entière de l'édifice en vertu de la loi [...] le pouvoir de délivrer les autorisations pour la tenue de toutes manifestations 
compatibles avec le caractère du lieu ». Enfin, la circulaire du ministère de la Culture du 7 juillet 1998 opère un retour aux principes de la gestion domaniale au sens où il est rappelé que « toute utilisation privative du domaine de l'État, y compris les cathédrales, donne lieu à la délivrance d'une autorisation et que cette compétence, pour les monuments inclus dans la liste annexée à la présente convention, relève de la seule Caisse nationale des monuments historiques et des sites ". II est toutefois précisé que « pour les édifices affectés au culte, l'accord du clergé affectataire doit être obtenu préalablement à toute manifestation non cultuelle ». L'autorisation des manifestations étrangères à l'affectation cultuelle des cathédrales apparaît donc partagée entre le Centre des monuments nationaux, autorité gestionnaire, et l'affectataire. Quelques années auparavant, le juge administratif avait lui aussi eu recours à cette solution de l'accord préalable de l'affectataire, s'agissant de l'organisation des visites d'un édifice cultuel monument historique, dans l'important arrêt Abbé Chalumey ${ }^{38}$.

Des difficultés subsistaient cependant, sur un plan financier pour l'essentiel et le recours à la loi avait été annoncé dès $2003^{39}$. II s'agissait d'une nécessité, essentiellement parce que la perception de droits de visite dans les édifices cultuels monuments historiques manquait de base légale (Gaillard, 2002 : 81). Les dispositions de l'article L. 2124-31 ont alors été insérées dans le Code de la propriété des personnes publiques, adopté en avril 2006. Le texte n'innove pas réellement dans la mesure où il entérine la solution infra-réglementaire et jurisprudentielle de l'accord préalable de l'affectataire religieux à tout usage non cultuel de l'édifice. Mais s'agissant de la perception des droits de visite et de la redevance domaniale perçue à l'occasion de manifestations culturelles dans les édifices du culte, il a le mérite de légaliser des pratiques devenues tout simplement inévitables, même si tout n'est pas réglé pour autant.

Ce texte est lui-même le fruit de négociations, qui ont duré plusieurs années. Alors qu'un texte de valeur législative est souvent synonyme de sécurité et de durabilité aux yeux des parties, les ambiguïtés qui affectent la version finale laissent cependant penser qu'il a été précédé d'une recherche de compromis qui n'a que partiellement abouti. La terminologie employée est empreinte d'une certaine maladresse - pour ne pas dire qu'elle est erronée (Untermaier, 2006) - mais, surtout, la mise en œuvre concrète de ces dispositions législatives pourrait être déjà dépassée. 


\section{De l'unilatéralité à la conventionalité ?}

Qu'il s'agisse de l'organisation matérielle des visites et manifestations culturelles ou de la perception des sommes auxquelles elles donnent lieu, le droit applicable issu du Code général de la propriété des personnes publiques a été ultérieurement enrichi, mais suivant deux directions assez divergentes, révélant la difficulté d'intervenir sur ce terrain sensible. On observe d'un côté une tendance du juge à encourager la voie conventionnelle pour dépasser les tensions potentielles entre logique culturelle et usage religieux, de l'autre, un recours à la négociation entre le ministère de la Culture et l'Église catholique qui s'est avéré nécessaire en dépit de l'adoption du texte de loi.

La voie conventionnelle encouragée. On fera remarquer que le recours à la convention figurait dans une version initiale du texte du code ${ }^{40}$, selon laquelle « une convention conclue entre la personne publique propriétaire et l'association cultuelle définit après avis du desservant, les modalités de la visite ou de l'usage ainsi que les règles relatives à la perception des droits et à la répartition de leur produit ». La rédaction finalement retenue de l'article L. 2124-31 CGPPP maintient une logique unilatérale et fait de ce texte une étape sur le point d'être dépassée lorsqu'elle est mise en perspective avec la jurisprudence ultérieure. Dans ce cadre, non seulement la logique qui transparaît est bien celle d'une contractualisation des relations entre les pouvoirs publics et l'Église en matière de patrimoine religieux, mais le principe central de l'accord préalable de l'affectataire est en outre revisité. Le contentieux est rare dans ce domaine et le juge administratif n'a pas encore été saisi de litiges nés postérieurement à 2006. Ce n'est donc pas à partir des dispositions du code que le juge administratif a orienté sa jurisprudence dans le sens d'une conventionalité des relations entre l'État et l'Église catholique. Bien que non directement reliées à l'organisation de pratiques culturelles et à la perception de droits de visites, deux espèces récentes renvoient à cette logique contractuelle applicable de manière générale à la valorisation du patrimoine religieux. Plus précisément, le juge administratif s'est prononcé sur la question de la légalité du financement public d'aménagements à des fins culturelles dans des édifices du culte. Le premier litige concernait l'installation d'un orgue dans l'église par la commune propriétaire, afin d'être utilisé par elle « dans le cadre de sa politique culturelle et éducative et, le cas échéant, par le desservant, pour accompagner l'exercice du culte ». L'unité de lieu pouvant 
se traduire par une commune utilisation de l'instrument, la Haute juridiction préconise que « des engagements soient pris afin de garantir une utilisation de l'orgue par la commune conforme à ses besoins et une participation de l'affectataire ou du propriétaire de l'édifice, dont le montant soit proportionné à l'utilisation qu'il pourra faire de l'orgue afin d'exclure toute libéralité et, par suite, toute aide à un culte» et que ces engagements «peuvent notamment prendre la forme d'une convention ${ }^{41}$. L'invitation est réitérée dans la seconde espèce, qui impliquait le financement d'un ascenseur permettant l'accès des visiteurs à la basilique lyonnaise de Fourvière. Là encore, la légalité de la subvention accordée sur le fondement d'un intérêt public local repose sur la condition « lorsque la collectivité territoriale accorde une subvention pour le financement des travaux, que soit garanti, notamment par voie contractuelle, que cette participation n'est pas versée à une association cultuelle et qu'elle est exclusivement affectée au financement du projet ${ }^{42}$.

Mais la jurisprudence la plus significative date de juin 2012, qui prend une certaine distance avec le principe de l'accord préalable de l'affectataire ${ }^{43}$, considéré jusqu'à présent par le Conseil d'État comme une prérogative de l'affectataire découlant de son pouvoir de réglementation des pratiques religieuses dans l'édifice. Or, le Conseil d'État considère dans cette affaire qu'à partir du moment où les visites en cause se déroulaient sur une partie de l'édifice (le toit-terrasse) qui n'est pas utilisée pour le culte, elles échappent au pouvoir de réglementation du ministre du culte... La commune recouvre ainsi un pouvoir de réglementation de l'édifice, en tant que propriétaire d'un bien classé monument historique et qu'elle entend valoriser. En outre, le Conseil d'État opère un renversement des responsabilités à l'égard des conditions de déroulement du culte. II revient en effet à la commune de « veiller à ce que les modalités d'organisation [des visites] ne conduisent pas à perturber l'exercice du culte à l'intérieur de l'édifice et soient compatibles avec l'affectation de l'édifice sur lequel les aménagements visités sont situés ».

Si ce dernier arrêt ne s'inscrit pas précisément dans la tendance à encourager une convention entre les parties, il est révélateur des insuffisances des dispositions de l'article L. 2124-31 CGPPP, qui ont conduit par ailleurs le ministère de la Culture et l'Église catholique à réagir par textes interposés pour en négocier les conditions concrètes d'application. 
Une loi négociée ? Dans un premier temps, et assez classiquement, ces dispositions de l'article L. 2124-31 CGPPP ont été suivies d'une circulaire interministérielle (ministères de la Culture et de l'Intérieur) du 21 avril $2008^{44}$. Le texte se contente d'une reprise assez laconique du droit en vigueur exposé dix ans auparavant dans la circulaire de 1998. Y figure également une explication sur les recettes et redevances susceptibles d'être perçues à l'occasion des visites et manifestations culturelles se déroulant dans les cathédrales ${ }^{45}$ et susceptibles, selon le texte du code, d'être partagées entre propriétaire public et affectataire cultuel. Ce détail n'a pas échappé à l'Église catholique qui a elle-même produit un texte (dénommé également « circulaire », n4-2009, du $1^{\mathrm{er}}$ mars 2009). II ne s'agit évidemment pas de les mettre sur le même plan. En revanche, cette dernière note mérite d'être mentionnée en ce qu'elle traduit le positionnement de l'Église catholique sur ces questions et illustre l'ambiguïté des dispositions du Code général de la propriété des personnes publiques. En effet, la terminologie employée laisse le lecteur au milieu du gué : le code se réfère classiquement à l'accord de l'affectataire pour tout usage non religieux, mais poursuit en disposant que « l'accord précise les conditions et les modalités de cet accès ou de cette utilisation », évoquant l'idée d'une négociation et d'un contrat entre les parties. Or, c'est précisément cette interprétation que réfute, cette fois sans ambiguïté, la note de la Conférence des évêques de France : «à propos de l'accord de l'affectataire, on utilisera plutôt le terme d'autorisation (qui renvoie à la dimension unilatérale) et on évitera d'employer le terme de convention (qui renvoie à une relation bilatérale) ». Cette remarque permet d'éclairer ce qui apparaît à première vue comme une acceptation par les autorités religieuses catholiques de cette méthode de gestion négociée des lieux de culte. En effet, en annexe de cette « circulaire " figure un modèle de texte intitulé Protocole d'accord avec la commune assorti de l'autorisation de l'affectataire. Utilisation d'une église communale pour des manifestations culturelles. Mais ce qui est proposé n'a que l'apparence d'une négociation et d'un accord bilatéral dans la mesure où le contenu est finalement défini unilatéralement, laissant une marge de manœuvre réduite aux personnes publiques propriétaires, en l'occurrence les communes.

Le passage des dispositions législatives précitées au mode contractuel entre l'État et l'Église catholique n'est pas encore un processus achevé. En effet, ni les différents textes précités ni la jurisprudence n'ont épuisé la nécessité pour les parties en présence de se concerter, en ce qui 
concerne les cathédrales pour le moment. Plusieurs réunions organisées par le ministère de la Culture ont donné lieu à la rédaction de «fiches » de travail élaborées conjointement avec les représentants de l'Église catholique ${ }^{46}$. La première porte sur les questions - relativement consensuelles - de sécurité dans les cathédrales. Mais elle ouvre la voie aux discussions sur la question plus délicate des redevances perçues (et de leur partage...) pour les visites et les manifestations culturelles dans ces lieux de culte.

Au final, il résulte de cette régulation un puzzle juridique fait de pièces hétérogènes sur le plan des sources, mais révélateur de l'impossibilité de s'en tenir à une intervention législative unilatérale.

Ce détour à travers l'exemple de la valorisation du patrimoine religieux montre que, plutôt que les normes juridiques, ce sont à la fois la négociation et la jurisprudence qui semblent les plus susceptibles d'introduire la souplesse nécessaire à l'adaptation d'un corpus légal souvent ancien et de modifier les équilibres institutionnels existants pour répondre aux exigences nouvelles d'une société pluraliste. Si le recours à la négociation comme outil d'ajustement inscrit le droit des religions dans le champ de la soft law, l'évolution de celui-ci face aux enjeux contemporains relève également d'une jurisprudence qui demeure fournie. Depuis plus d'un siècle déjà, ce sont principalement les juridictions administratives qui sont à l'origine de décisions sur les questions religieuses pour préciser les règles relatives à l'organisation des confessions et à leurs relations avec les pouvoirs publics, qu'il s'agisse par exemple de se prononcer sur les pouvoirs de police du maire en matière de manifestations extérieures du culte ${ }^{47}$, sur la neutralité religieuse des agents publics ${ }^{48}$ ou récemment encore sur le financement public des cultes $^{49}$. Si les juridictions judiciaires n'ont quant à elles jamais été totalement absentes du champ contentieux lié au fait religieux, elles développent depuis quelques décennies une jurisprudence plus particulièrement centrée sur l'expression des convictions religieuses des individus et l'encadrement de la liberté individuelle de religion, cette évolution dessinant progressivement de nouveaux contours au fait religieux saisi par diverses branches du droit (droit pénal, droit du travail, droit de la famille...). Une analyse exhaustive de cette jurisprudence reste à mener, mais on peut d'ores et déjà considérer que «le droit des religions, qui était caractérisé en France jusqu'à une période récente essentiellement par la question des relations avec l'État, est de plus en plus concerné 
par des problèmes de relations entre individus ou entre groupes » (Messner et al., 2013 : 24). Par ailleurs, la montée en puissance des droits fondamentaux - notamment à travers l'influence de la jurisprudence de la Cour européenne des droits de l'homme - et le développement du contrôle de proportionnalité par le juge ${ }^{50}$ conduisent à une plus grande prise en compte des droits de l'individu et des relations interindividuelles. Alors que le principe de laïcité étend une emprise symbolique et politique qui tend à cristalliser les positions acquises, c'est aujourd'hui l'interprétation de la règle de droit plutôt que son adaptation ou sa négociation qui paraît le mieux à même d'assurer la pleine application du principe de liberté de religion.

\section{Notes}

${ }^{1}$ Expression utilisée dans un souci d'objectivation et renvoyant à la fois à la dimension de fait collectif et matériel et de fait symbolique et sensible (Borne et Willaime, $2007: 37$ ).

${ }^{2}$ Sur ce thème, voir notamment Association Henri Capitant, 2009 et Fornerod (2014).

${ }^{3}$ Loi $n^{\circ}$ 2001-504 du 12 juin 2001 tendant à renforcer la prévention et la répression des mouvements sectaires portant atteinte aux droits de l'homme et aux libertés fondamentales; Loi n²004-228 du 15 mars 2004 encadrant, en application du principe de laïcité, le port de signes ou de tenues manifestant une appartenance religieuse dans les écoles, collèges et lycées publics; Loi n²010-1192 du 11 octobre 2010 interdisant la dissimulation du visage dans l'espace public.

${ }^{4}$ Corpus évalué à environ deux cents dispositions (textes ou extraits de textes), voir Curtit, 2014.

${ }^{5}$ Les dispositions juridiques en matière de non-discrimination sont introduites en droit français dans les années 2000, notamment sous l'impulsion du droit de l'Union européenne.

${ }^{6}$ Le principe de non-subventionnement public des activités religieuses introduit par la loi du 9 décembre 1905 connaît en effet de nombreuses exceptions.

${ }^{7}$ Art. 1 : « La République assure la liberté de conscience. Elle garantit le libre exercice des cultes sous les seules restrictions édictées ci-après dans l'intérêt de l'ordre public. »; Art. 2 : « La République ne reconnaît, ne salarie ni ne subventionne aucun culte [...]. »

${ }^{8}$ Loi n $2004-228$ du 15 mars 2004 encadrant, en application du principe de laïcité, le port de signes ou de tenues manifestant une appartenance religieuse dans les écoles, collèges et lycées publics.

${ }^{9}$ Loi n 2010-1192 du 11 octobre 2010 interdisant la dissimulation du visage dans l'espace public. 
10 Voir également la volonté récente des acteurs politiques français de légiférer pour étendre la neutralité religieuse aux entreprises et associations privées.

${ }^{11}$ Art. 10 de la Déclaration française des droits de l'homme de 1789 : « Nul ne doit être inquiété pour ses opinions, même religieuses, pourvu que leur manifestation ne trouble pas l'ordre public. »; Art. 1 de la Constitution de 1958 : «La France est une République indivisible, laïque, démocratique et sociale. Elle assure l'égalité devant la loi de tous les citoyens sans distinction d'origine, de race ou de religion. Elle respecte toutes les croyances [...] »; Art. 1 de la loi du 9 décembre 1905: «La République assure la liberté de conscience. Elle garantit le libre exercice des cultes sous les seules restrictions édictées ci-après dans l'intérêt de l'ordre public. 》

${ }^{12}$ Avec de très rares exceptions, voir notamment la loi $\mathrm{n}^{\circ} 78-4$ du 2 janvier 1978 relative aux régimes d'assurance maladie, maternité, invalidité, vieillesse, applicables aux ministres des cultes et membres des congrégations et collectivités religieuses.

${ }^{13}$ Consulter la liste des textes sur www.legirel.cnrs.fr

${ }^{14}$ Voir par ex. la circulaire du 18 mai 2004 sur le port de signes religieux dans les établissements d'enseignement publics pour la mise en œuvre de la loi 15 mars 2004, ou les six circulaires adressées à diverses autorités administratives et judiciaires qui présentent la loi du 11 octobre 2010 interdisant la dissimulation du visage dans l'espace public.

${ }^{15}$ Voir par ex. : circulaire du 23 juin 2010 sur le support institutionnel de l'exercice du culte ; circulaire du 29 juillet 2011 sur les édifices du culte : propriété, construction, réparation et entretien, règles d'urbanisme, fiscalité ; circulaire du 25 août 2011 sur la réglementation des cultes outre-mer.

${ }^{16}$ Circulaire du 16 août 2011 sur le rappel des règles afférentes au principe de laïcité : demandes de régimes alimentaires particuliers dans les services de restauration collective du service public.

${ }^{17}$ Circulaire du 29 juillet 2011 précitée.

${ }^{18}$ Circulaire du 23 juin 2010 précitée.

${ }^{19}$ Circulaire du 2 février 2005 relative à la laïcité dans les établissements de santé.

${ }^{20}$ Circulaire du 16 août 2011 précitée

${ }^{21}$ Par ailleurs, l'article L. 2213-9 du Code général des collectivités territoriales, issu de la loi municipale du 5 avril 1884, précise que les pouvoirs de police du maire concernant notamment les inhumations et les exhumations doivent être accomplis « sans qu'il soit permis d'établir des distinctions ou des prescriptions particulières à raison des croyances ou du culte du défunt ou des circonstances qui ont accompagné sa mort ».

${ }^{22}$ Circulaire $n^{\circ} 75-603$ du 28 novembre 1975 sur l'inhumation des Français de confession islamique. 
${ }^{23}$ Possibilité prévue alors essentiellement pour les harkis, anciens supplétifs de l'armée française pendant la guerre d'Algérie.

${ }^{24}$ Circulaire INT/A/08/00038/C du 19 février 2008 sur la police des lieux de sépulture.

25 « La soft law [...] contient en effet en principe des énoncés à la normativité non juridique qui favorisent le conseil, l'invitation et plus généralement la recommandation » (Lavergne, 2011 : 287).

${ }^{26}$ Voire d'un « droit souterrain », selon l'expression appliquée aux circulaires dans le rapport public 2006 du Conseil d'État.

27 «L'institution de carrés confessionnels dans les cimetières n'est donc pas possible en droit. Toutefois, en pratique, les carrés confessionnels sont admis et même encouragés par les pouvoirs publics afin de répondre aux demandes des familles, de confession musulmane notamment, de voir se créer dans les cimetières des lieux d'inhumation réservés à leurs membres. »(Conseil d'État, $2004:$ 326-327).

${ }^{28}$ Voir par ex. les vives réactions des acteurs sociopolitiques à la suite de l'arrêt Baby Loup (Cass. soc., 19 mars 2013, $\left.n^{\circ} 11-28.845\right)$ qui démontrent pour le moins une méconnaissance du périmètre d'application du principe de laïcité.

${ }^{29}$ II est intéressant cependant de noter que ce refus était contraire « au vœu de la majorité de l'épiscopat de France » (Méjan, $1973: 271)$.

${ }^{30}$ Loi du 2 janvier 1907 concernant l'exercice public des cultes et loi du 13 avril 1908 modifiant la loi du 9 décembre 1905 sur la séparation des Églises et de l'État.

${ }^{31}$ Commentaire de Jean Rivero à propos de la loi du 2 janvier 1907.

${ }^{32}$ Sur le régime juridique du patrimoine religieux, voir Fornerod, 2013.

${ }^{33}$ Les 87 cathédrales appartiennent à l'État, tandis que les communes sont propriétaires de la quasi-totalité des églises construites avant 1905.

${ }^{34}$ Sur cette question des lieux de culte, voir Florès-Lonjou, 2001.

${ }^{35}$ Nous ne distinguerons pas ici entre l'accès au patrimoine religieux pour les diverses manifestations culturelles qui peuvent s'y dérouler et les visites des édifices cultuels comme monuments historiques, bien qu'une telle distinction puisse se déduire des textes et renvoie à des enjeux différents.

${ }^{36}$ Circulaire du 4 août 1969 du ministre d'État chargé des affaires culturelles aux conservateurs régionaux des bâtiments de France, relative aux autorisations de manifestations diverses, d'illumination et de tournage de films dans les monuments de l'État. Texte de la circulaire dans Dussaule, 1974 : 67-69. 
37 Circulaire $n^{\circ} 88-129$ du 27 avril 1988 concernant le régime de délivrance des autorisations d'occupation temporaire dans les cathédrales.

${ }^{38}$ CE Ass., 4 novembre 1994, Abbé Chalumey. Voir aussi CE ord., 25 août 2005, Commune de Massat, $n^{\circ} 284307$.

${ }^{39}$ Voir par ex. Lesegretain Claire, «Le dossier sensible des cathédrales », La Croix, 13 février 2003, p. 5 ; De Gaulmyn Isabelle et Schmidt Pierre, « Une loi précisera l'utilisation des cathédrales », La Croix, 21 mai 2003, p. 20.

${ }^{40}$ Voir Fornerod (2013 : 440). Voir aussi De Gaulmyn Isabelle et Schmidt Pierre, « Une loi précisera l'utilisation des cathédrales », La Croix, 21 mai 2003, p. 20. On peut lire dans ce dernier article qu' « une future loi devrait donner un cadre législatif à cette activité, et réaffirmer, ce qui était d'importance aux yeux des évêques, le strict respect de l'affectation d'abord cultuelle des édifices. Ensuite, des conventions entre les pouvoirs publics et l'évêque du lieu seront nécessaires pour définir plus précisément les conditions de perception des droits ».

${ }^{41}$ CE, 19 juillet 2011, Commune de Trélazé, n³08544.

${ }^{42}$ CE, 19 juillet 2011, Fédération de la libre pensée et de l'action sociale du Rhône et M. P., n 308817.

${ }^{43}$ CE, 20 juin 2012, Commune des Saintes-Maries-de-la-Mer, n 340648. « Considérant que l'affectation résultant de la combinaison des dispositions citées ci-dessus s'applique à l'ensemble d'un édifice cultuel, y compris ses dépendances nécessaires, fonctionnellement indissociables de l'édifice cultuel ; que, dès lors, la toiture d'un édifice cultuel, en tant qu'elle est nécessaire au bon déroulement des célébrations cultuelles organisées dans l'édifice qu'elle protège, est affectée au culte en vertu de ces mêmes dispositions ; que, toutefois, il en va autrement d'aménagements qui, alors même qu'ils sont situés sur le toit de l'édifice cultuel, doivent être regardés, compte tenu notamment de leurs caractéristiques propres et de la possibilité d'y accéder sans entrer dans l'édifice cultuel, comme fonctionnellement dissociables de cet édifice ; que la commune peut, sans avoir à recueillir l'accord préalable du desservant de l'église, organiser des visites de tels aménagements ; qu'il lui appartient de veiller à ce que les modalités d'organisation de celles-ci ne conduisent pas à perturber l'exercice du culte à l'intérieur de l'édifice et soient compatibles avec l'affectation de l'édifice sur lequel les aménagements visités sont situés. 》

${ }^{44}$ Circulaire interministérielle $n^{\circ} 2008 / 002$ du 21 avril 2008 relative à l'utilisation des édifices de culte appartenant à l'État à des fins non cultuelles.

${ }^{45}$ Selon le texte même de la circulaire, les principes rappelés « ont, par ailleurs, vocation à inspirer la pratique suivie pour les édifices cultuels appartenant à des collectivités territoriales ».

Studies in Religion / Sciences Religieuses 2016

Vol. 45(2) 111-126

DOI: $10.1177 / 0008429816636083$ 
${ }^{46}$ Nous remercions pour ces informations Madame Françoise Jeanmougin, adjointe au chef du Bureau du patrimoine immobilier, et Madame Judith Kagan, chef du Bureau de la conservation du patrimoine mobilier et instrumental du ministère de la Culture.

${ }^{47}$ CE, 19 février 1909, Abbé Olivier.

${ }^{48}$ CE, 3 mai 1950, Dlle Jamet.

${ }^{49}$ CE, 19 juillet. 2011 (5 espèces), concl. Geffray E. (2011). Revue française de droit administratif : 967-986.

${ }^{50}$ En matière religieuse, voir par ex. Cass. soc., 19 mars 2013, Baby Loup, n 11-28.845 ou au plan européen CEDH, 15 janvier 2013, Eweida et a.

\section{Bibliographie}

Association Henri Capitant (2009) Le droit souple. Paris : Dalloz, 2009.

Borne D et Willaime J.-P (2007) Enseigner les faits religieux : quels enjeux ? Paris : Armand Colin.

Chevallier J (2001) La régulation juridique en question. Droit et société, vol. n49, $3:$ 827-846.

Conseil d'État (2004) Un siècle de laïcité. Rapport public 2004. Paris : La Documentation française, coll. «Études et documents ».

Conseil d'État (2006) Sécurité juridique et complexité du droit. Rapport public 2006. Paris : La Documentation française, coll. «Études et documents ».

Curtit F (2014) Le droit français des religions au miroir des textes. In Droit et religion en Europe. Études en I'honneur de Francis Messner. Strasbourg: Presses universitaires de Strasbourg, p. 63-72.

Dussaule P (1974) La loi et le service des monuments historiques français. Paris : La Documentation française.

Florès-Lonjou M (2001) Les lieux de culte en France. Paris : Cerf.

Flores-Lonjou M (2011) Édifice cultuel - Droit français. In Dictionnaire Droit des religions. Paris : CNRS éd., pp. 243-245.

Fornerod A (2013) Le régime juridique du patrimoine religieux. Paris : L'Harmattan.

Fornerod A (2014) Droit des religions et soft law. In Droit et religion en Europe. Études en l'honneur de Francis Messner. Strasbourg : Presses universitaires de Strasbourg, p. 99-110.

Gaillard Y (2002) 51 mesures pour le patrimoine monumental. Rapport d'information, $\mathrm{n}^{\circ} 378$ (2001-2002). Paris : Sénat.

Lavergne B (2011) Recherche sur la soft law en droit public français. Thèse de droit public, Université Toulouse 1 Capitole. 
Méjan F (1973) De l'histoire et du droit actuel des édifices du culte. Bulletin de la Société de l'Histoire du Protestantisme français, t. $119: 248-286$.

Messner F, Prélot P.-H et Woehrling J.-M (2013) Droit français des religions. Paris : LexisNexis, $2^{\mathrm{e}}$ éd, coll. «Traités ».

Rivero J (1965) Histoire et prospective dans le statut des édifices du culte en régime de séparation. In Études d'histoire du droit canonique dédiées à Gabriel Le Bras, t. 1. Paris : Sirey, pp. 681-688.

Thibierge C (2003) Le droit souple. Réflexion sur les textures du droit. Revue trimestrielle de droit civil : 599-628. Untermaier E (2006) Les églises et le code général de la propriété des personnes publiques. À propos de l'article L. 2124-31. AJDA : 2210-2213. 